\title{
DIAGNOSIS RELACIONAL: UNA EVALUACIÓN DINÁMICA Y TRANSTEÓRICA ENFOCADA EN LA PERSONA A TRAVÉS DE LA RELACIÓN
}

\author{
RELATIONAL DIAGNOSIS: \\ A DYNAMIC AND TRANSTHEORETICAL ASSESSMENT \\ FOCUSED ON THE PERSON THROUGH RELATIONSHIP
}

\author{
Bibiana Martín Corcuera \\ Hogar Izarra, Comunidad Terapéutica Integrativa para Personas con Trastorno Mental, \\ Bilbao, Bizkaia, España \\ ORCID: https://orcid.org/0000-0001-7649-5298
}

Mar Gómez Masana

ARETÉ psicología-psicoterapia, Barcelona, España

ORCID: https://orcid.org/0000-0002-8818-0303

Cómo referenciar este artículo/How to reference this article:

Martín Corcuera, B. y Masana, M. (2021). Diagnosis relacional: Una evaluación dinámica y transteórica enfocada en la persona a través de la relación. Revista de Psicoterapia, 32(118), 25-45.

https://doi.org/10.33898/rdp.v32i118.483

\section{Resumen}

Partiendo del significado general del concepto de Diagnóstico, el presente trabajo se centra en el Psicodiagnóstico y, desde ahí, profundiza en la forma y el fondo de un psicodiagnóstico en el campo de la Psicoterapia Relacional Integrativa, cómo un modelo transteórico y transdiagnóstico. O lo que es lo mismo, la diagnosis relacional, como visión global de la persona, desde las dimensiones fisiológica, emocional, cognitiva, relacional, conductual y espiritual. En el desarrollo de este artículo, se revisa la historia de rechazo del concepto clásico de diagnóstico, desde diferentes escuelas psicoterapéuticas, entre ellas las Psicoterapias Humanistas. Se reflexiona sobre los efectos del diagnóstico en los clientes. $Y$ se describe, a modo de ejemplo, una forma particular de llevar a cabo, de poner en práctica, el proceso de Diagnosis Relacional.

Palabras clave: psicoterapia integrativa relacional, diagnosis relacional, psicodiagnóstico, transdiagnóstico, transteoría

\begin{abstract}
Starting from the general meaning of the concept of Diagnosis, this work focuses on Psychodiagnosis and, from there, delves into the form and substance of a psychodiagnosis in the field of Integrative Relational Psychotherapy, as a transtheoretical and transdiagnostic model. Or what is the same, relational diagnosis, as a global vision of the person, from the physiological, emotional, cognitive, relational, behavioral and spiritual dimensions. In the development of this article, the history of rejection of the classic diagnostic concept is reviewed, from different psychotherapeutic schools, among them Humanistic Psychotherapies. It reflects on the effects of the diagnosis on clients. And it describes, by way of example, a particular way of carrying out, of putting into practice, the process of Relational Diagnosis.

Keywords: integrative relational psychotherapy, relational diagnosis, psychodiagnosis, transdiagnosis, transtheory
\end{abstract}

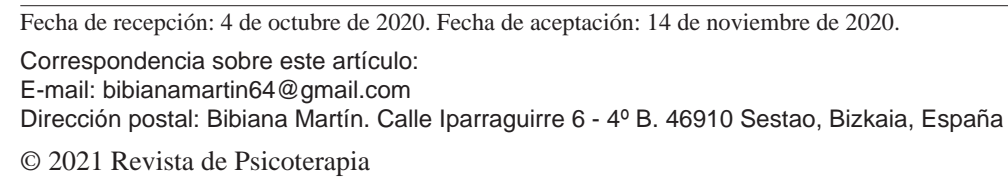


El concepto de diagnóstico es extensible a los más diversos campos. Hace referencia a la acción y efecto de diagnosticar, que "es el proceso de reconocimiento, análisis y evaluación de una cosa o situación para determinar sus tendencias, solucionar un problema o remediar un mal" (Significados.com).

En el campo de la Psiquiatría y Psicología el término Psicodiagnóstico, aparece por primera vez en la obra de H. Rorschach, publicada en 1921, con el título Psychodiagnostik. Este término tiene su origen etimológico en un compuesto de tres vocablos griegos: «psikhé», «diá» y «gignosko», que se puede traducir como conocer a fondo el alma o conocer a fondo la mente (Wikipedia).

Un psicodiagnóstico es un proceso de evaluación en el que a través de la recogida de información por diferentes vías (entrevistas, autoinformes, observación directa, registros, test o técnicas proyectivas, información de personas de su entorno...), se evalúan las distintas áreas del funcionamiento psicológico de un sujeto (fisiológica, afectiva, cognitiva, conductual y relacional), y se intenta definir su estado de salud mental, las características de su personalidad y conflictos que subyacen al motivo de consulta que la persona trae consigo, con la finalidad de alcanzar un objetivo (selección de personal, orientación, cambiar comportamientos, etc.).

Dependiendo del modelo teórico desde el que se sitúe el profesional se enfocará en recoger, principalmente, una información u otra:

a. Modelo psicométrico: Rasgos de personalidad.

b. Modelo médico: Enfermedades mentales. La identificación de la naturaleza de una enfermedad mediante pruebas y la observación de sus signos o síntomas.

c. Modelo psicodinámico: Mecanismos internos, lo inconsciente.

d. Modelo cognitivo conductual: Conductas.

e. Modelo integrativo relacional: Funcionamiento específico del individuo en todas sus dimensiones interrelacionadas.

Si bien el psicodiagnóstico puede realizarse con estilos y duraciones variables, su objetivo principal será lograr una comprensión profunda y completa de la personalidad total del cliente, y resulta fundamental para una orientación del plan de tratamiento, a ajustar según el proceso dinámico activo.

Conviene recordar, que al representar el psicodiagnóstico una "foto" de la personalidad, sus conclusiones son válidas para el "aquí y ahora” de ese sujeto dado, esto es: la personalidad es dinámica y los resultados obtenidos por una misma persona pueden variar a lo largo del tiempo.

Por eso, aunque convencionalmente, esta psicodiagnosis (inicial) tiene distintas etapas -pre-entrevista, entrevista psicodiagnóstica, batería psicodiagnóstica, informe clínico-, en realidad, la evaluación clínica, es un proceso dinámico y continuo, puesto que la persona y sus circunstancias son cambiantes, van evolucionando.

En esta línea, la Psicoterapia Relacional Integrativa propone, en su Teoría de los Métodos, mantener una actitud de interés y apertura en acompañar al cliente al descubrimiento de sí mismo en todas las dimensiones, sin dar por hecho que el 
terapeuta conoce lo que le pasa al sujeto. De esta manera, el terapeuta se encontrará “evaluando" (recogiendo información, haciendo y contrastando hipótesis...) a lo largo de todo el proceso de tratamiento y de la relación terapéutica.

En Psicoterapia Integrativa, nuestra indagación empieza con la suposición de que el terapeuta no sabe nada sobre la experiencia subjetiva del cliente (...) "El propósito final de la indagación es ayudar a los clientes a descubrir los modos en que han aprendido a interrumpir el contacto interno y el contacto externo (...)" (Erskine et al., 1999/2012, p. 56).

\section{El Psicodiagnóstico y las Psicoterapias Humanistas}

Desde una visión retrospectiva, "durante varias décadas la Psicoterapia de la Gestalt, al igual que el resto de las Psicoterapias Humanistas, consideró que el psicodiagnóstico era malsano por el etiquetaje rígido que comportaba, prescindiendo de lo idiosincrático" (Gimeno-Bayón, 2012, p. 146).

Tal y como recoge Gimeno-Bayón(2012), Sugarman(1987) realizó una síntesis de la crítica al psicodiagnóstico desde las Psicoterapias Humanistas, considerando la evaluación reduccionista, artificial, sin prestar atención a la relación entre terapeuta y paciente, juzgando al paciente y resultando demasiado intelectual. Pero, con el paso del tiempo, "El psicodiagnóstico fue evolucionando por diferentes factores, entre ellos las críticas recibidas. El mismo autor (Sugarman) defiende que hoy en día las críticas mencionadas solo se justifican en evaluadores poco sofisticados" (Gimeno-Bayón, 2012, p. 148).

Continuando con Gimeno-Bayón (2012), para Fagan (1973) "el psicodiagnóstico es la observación e identificación de las estructuras o patrones recurrentes de una persona a la hora de enfrentar los problemas, algunos de esos patrones que tiende a utilizar son disfuncionales, por su cronicidad o anacronismo" (Gimeno, 2012, p. 147).

El diagnóstico es inevitable, aunque algunas corrientes teóricas lo disfracen con diferentes terminologías. En modelos teóricos como la Psicoterapia de la Gestalt y el Análisis Transaccional, por ejemplo, tras el rechazo inicial del psicodiagnóstico convencional, recurrieron a traducir los términos del psicodiagnóstico clásico al vocabulario de su propio modelo, para facilitar el diálogo con los profesionales ajenos al mismo.

Es inevitable, porque los seres humanos necesitan darle un significado a la experiencia. En la relación terapéutica, tanto cliente como terapeuta están comprometidos en la comprensión del proceso del cliente y el psicodiagnóstico fenomenológico puede resultar una herramienta, un lenguaje comprensivo de los procesos del cliente y para el cliente. Por consiguiente, el interrogante no sería si diagnóstico sí o diagnóstico no, sino cómo realizarlo.

No cabe duda, que la tarea diagnóstica tiene también sus aspectos positivos, que resulta interesante reseñar.

Desde la perspectiva del cliente, el poner un nombre a lo que está experimen- 
tando le ayuda a darle sentido y, con frecuencia, el sentir que el otro le comprende y puede entender por lo que está pasando y cómo ayudarle en sus dificultades y vulnerabilidades, le ofrece cierta sensación de seguridad. Una muestra de ello son los testimonios de tantas y tantas personas con algún trastorno psicosomático (fibromialgia...), que hasta que no pudieron poner nombre a sus dolencias y se sintieron reconocidas y entendidas por un profesional confiable, vivieron su sintomatología, como una experiencia vergonzante, culpabilizadora y traumática.

Desde la perspectiva del terapeuta, un buen diagnóstico le ayuda a orientar a su cliente: le ayuda a planificar el tratamiento, cuando éste es preciso, a elegir los métodos y las técnicas de intervención y adaptarlas a la persona. El hablar en un lenguaje común facilita el entendimiento y simplifica la transmisión de información de unos profesionales a otros. Ofreciendo al cliente la sensación de un proceso continuo cuando tiene que pasar por diferentes profesionales. en lugar de vivirlo como experiencias independientes y desconectadas, que en absoluto fomentan la integración personal.

Entre las observaciones realizadas por las autoras desde su experiencia clínica, respecto al efecto del psicodiagnóstico, tanto en personas con trastorno psicológico como en aquellas personas que consultan por circunstancias o crisis vitales (problemas en el trabajo, en la pareja, síndrome de nido vacío, relación con los padres...) destacarían:

a) Algunos clientes se sienten asustados, desconcertados, perdidos y muy vulnerables ante las manifestaciones de guion (o síntomas) que presentan y necesitan que les ayuden a poner nombre y a encontrar el sentido a lo que les está aconteciendo, o confirmar el sentido que ellos han dado, con otra persona que perciben como confiable y más sabia o experta, sobre todo en etapas iniciales de consulta.

b) Para otros, el diagnóstico puede resultar limitador, por el riesgo de que el sujeto se identifique con la etiqueta del mismo y comprometa su evolución, renunciando a su auténtico yo y viviendo una vida sobreadaptada. En estos casos, a través de la experiencia contratransferencial, el terapeuta puede percibir la sensación de estar ante alguien artificial, vacío de contenidos propios, que repite estereotipos o definiciones ajenas sobre quiénes son.

c) La connotación negativa de la etiqueta y el estigma social, en ocasiones, conlleva que algunos sujetos no acepten el diagnóstico y adopten una actitud de pelea (lucha interna y/o externa) o negación, que les impide asumir su vulnerabilidad e implicarse en su tratamiento. Una forma de manejar esta situación, para facilitar la implicación del interesado, es proponer al cliente la intención de dejar de lado la etiqueta y de enfocarse en hablar de las dificultades que presenta y cómo le afectan en su vida cotidiana y en su proyecto de futuro.

d) No faltan los que sacan beneficio secundario a la etiqueta y la utilizan de justificación para hacer lo que les apetece, para recibir ayudas económicas o para no asumir sus responsabilidades. En estos casos, el estigma social que se suele denunciar es más un autoestigma. 
e) En ocasiones, como se recoge desde la visión sistémica (Linares et al., 2001), asumir el rol de enfermo permite mantener la homeostasis al sistema familiar. Y no resulta extraño ser testigos de que cuando el paciente designado mejora, algún otro miembro de la familia empeora o comienzan a surgir conflictos relacionales o bien, de alguna manera, empiezan a boicotear el tratamiento de nuestro cliente.

f) El tener una impresión diagnóstica permite al terapeuta orientarse sobre cómo relacionarse con una persona concreta, facilita el establecimiento del vínculo y puede evitar errores. Por ejemplo, ante un cliente con un estilo o trastorno paranoide, le permite calcular acertadamente la distancia que necesita el otro para no sentirse invadido y amenazado y facilita al terapeuta la posibilidad de ofrecer una respuesta emocional recíproca sintónica.

g) También, la comprensión de los procesos en los que está inmersa facilita que la persona se haga cargo de sí misma, empoderándose y responsabilizándose más de su propio tratamiento. Contando así, el terapeuta, con un aliado en el proceso terapéutico.

h) En ocasiones, los diagnósticos que se incluyen en el informe clínico de un cliente, más que ayudar a conocerlo y entenderlo, desorientan. Bien porque no son acertados, porque se asignaron en un momento determinado y no se han vuelto a revisar, porque en algunos informes se añaden tantos diagnósticos que confunden...

\section{Diagnóstico en la Psicoterapia Relacional Integrativa}

Si algo ha quedado claro de estos años de controversias es que, ya no es útil el diagnóstico desde las categorías del DSM, en sus diferentes versiones, incluida la última (5 ed.; DSM-5; American Psychiatric Association, 2014) y la Clasificación Internacional de Enfermedades (10 ed.; CIE-10; Organización Mundial de la Salud, 2010) que suelen complicar más que facilitan la tarea del terapeuta.

Desde esta realidad surge el movimiento transdiagnóstico y transteórico con autores como Livesley (2018), que plantea la importancia de un cambio de mirada para centrarse en el enfoque de factores comunes para la integración en psicoterapia.

Dado que estos mecanismos representan la mayor proporción de cambios en los resultados, existen poderosas razones para hacer de estos mecanismos la base de la terapia transteórica. Esta idea parece especialmente pertinente para tratar el trastorno de personalidad, porque el especial énfasis en los factores relacionales que conlleva un enfoque de factores comunes resulta especialmente pertinente para tratar un trastorno que se caracteriza por una disfunción interpersonal crónica (Livesley, Jackson, y Jang, 1994; Livesley, 1998, 2003b). (Livesley, 2018, p. 97)

E incluso, más allá de los factores comunes, la integración teórica es de suma importancia si realmente se quiere dar una atención psicoterapéutica que ayude al cliente a la comprensión en profundidad del sí mismo, de sus relaciones y del mundo, tal y como señala Mirapeix (2018), otro de los representantes del movimiento transteórico y transdiagnótico. 
En esta dirección de mi visión centrada en la personalidad y, por ende, en el self y las relaciones interpersonales, subyace una concepción puramente transdiagnóstica sustentada en la detección e intervención focal sobre mecanismos transdiagnósticos. Estos, siendo importantes como lo son, adquieren su auténtica dimensión ideográfica cuando son referidos a la identidad del individuo y al como éste se relaciona con los demás y con el mundo. (Mirapeix, 2018, p. 112)

En esta línea, la propuesta de la Psicoterapia Relacional Integrativa, desde la visión de las autoras, encajaría en la búsqueda de la coherencia entre transdiagnóstico, transteoría y transmetodología. Una coherencia que también se puede identificar en otras Psicoterapias Humanistas, como por ejemplo la Psicoterapia Integradora Humanista desarrollada por Gimeno-Bayón y Rosal (2001).

Para aproximarse al concepto de diagnóstico, tal y como se entiende desde la Psicoterapia Relacional Integrativa, conviene tener en cuenta el significado de la palabra integrativa, que constituye la esencia de este modelo teórico.

Tal y como señalan Erskine y Moursund (1988/2014), el término “integrativo" se refiere tanto a la síntesis de teorías y métodos de la psicoterapia a nivel fisiológico, afectivo, cognitivo y conductual, así como, al resultado de la psicoterapia: la integración o la asimilación en el interior de la persona de los aspectos de su personalidad fragmentados o fijados. Es la integración de los diferentes modelos teóricos, de una forma coherente y consistente lo que permite que el enfoque Relacional Integrativo sea un sistema comprensivo del ser humano.

El modelo de la Psicoterapia Relacional Integrativa (Erskine, 1997; Erskine, 2010; Erskine, 2015; Erskine y Moursund, 1988/2014; Erskine et al., 1999/2012; Moursund y Erskine, 2004; O’Reilly-Knapp y Erskine, 2003), se centra en las características humanas únicas y el valor único de cada individuo. Incorporando dentro de su enfoque, las aportaciones de múltiples teorías, es decir, un modelo transteórico: el concepto de Autorrealización y Autoactualización de Abraham Maslow (las personas están motivadas por la necesidad de convertirse en lo mejor que pueden llegar a ser); la Psicoterapia Centrada en el Cliente de Carl Rogers (cuya cura consiste en restaurar las condiciones de crecimiento: comprensión, aceptación incondicional y autenticidad en la relación); los conceptos y el modo del trabajo emocional de la Psicoterapia de la Gestalt, de Fritz y Laura Perls (la calidad del contacto en las relaciones humanas. La capacidad para el contacto, es salud, la ausencia de dicha capacidad, es no-salud); los conceptos del modelo del Análisis Transaccional de Eric Berne (la forma en que las personas estructuran sus identidades -sus distintos yoes-, las transacciones -la comunicación- entre las personas y por su manera de organizar el curso de sus vidas, o lo que es lo mismo, el guion de Vida); las perspectivas psicoanalíticas contemporáneas -la Psicología del Self de Heinz Kohut, la escuela británica de psicoanálisis de las Relaciones Objetales (la necesidad de relaciones en la vida y quéle ocurre a la persona cuando esas relaciones no funcionan de acuerdo a sus necesidades) y la perspectiva intersubjetiva en el 
psicoanálisis; las teorías e investigación sobre el desarrollo humano centrado en el desarrollo evolutivo infantil de Piaget, Erickson, Bowlby, Margaret Mahler, Daniel Stern (ayudan a entender en qué Estado del Yo y en qué edad está una persona); conceptos de la Terapia Conductual, para la parte más funcional; y la atención al cuerpo y el trabajo corporal de los modelos de las Psicoterapias Corporales.

Una vez aclarada su naturaleza transteórica y transmetodológica, para continuar explorando el concepto de psicodiagnóstico, conviene revisar los principios filosóficos en los que este modelo basa su teoría (Erskine, 2016) y, a su vez, de forma congruente, representan una guía para la práctica clínica.

Erskine (2015/2016), propone 8 principios filosóficos que influyen en la perspectiva, la actitud y en las interacciones del terapeuta con los clientes:

1. Todas las personas son igualmente valiosas; de forma que al establecer un diagnóstico hay que evitar que se convierta en un acto de poder o de descuento.

2. Toda experiencia humana se organiza fisiológica, afectiva y/o cognitivamente; es importante observar a la persona en su globalidad.

3. Toda conducta humana tiene significado en algún contexto. Es importante mantener una perspectiva evolutiva que ayude a entender síntomas, comportamientos, actitudes..., que tal vez en el hoy no tengan sentido, pero en otra etapa evolutiva sí.

4. El contacto interno y externo es esencial para el funcionamiento humano, por lo que las alteraciones, en este sentido, orientan a la hora de elaborar las impresiones diagnósticas.

5. Todas las personas buscan relaciones y son interdependientes a lo largo de la vida; la cantidad y la cualidad de estas ofrecen una información valiosa para la tarea diagnóstica.

6. Los seres humanos tienen el impulso innato de crecer y desarrollarse; uno de los peligros del diagnóstico es que el individuo adopte como su identidad propia e inamovible la foto estática, el reflejo de sí, que le muestra y quede estancado su desarrollo personal.

7. Los seres humanos sufren perturbaciones relacionales, no "psicopatología".

8. El proceso intersubjetivo en psicoterapia es más importante que el contenido de la psicoterapia.

De todos ellos, hay dos principios que se encuentran especialmente conectados con el concepto de la diagnosis relacional, porque conducen a replantearse lo patológico y la normalidad.

El primero: “Toda conducta humana tiene significado en algún contexto". Si se acepta que todas las personas tienden a buscar estructura, a organizar y dar sentido a la experiencia (Berne, 1973/1974), resulta coherente deducir que cualquier conducta, desde las más razonables o esperables a los comportamientos más extraños y problemáticos, tienen un significado en algún contexto, presente o histórico, aunque el terapeuta, y en ocasiones su cliente, no conozcan ese significado. 
Si se asume que esto es así, cualquier conducta o cualquier síntoma sería una reacción natural de supervivencia, y un intento adaptativo en el contexto en que se adoptó inicialmente. Por ejemplo, para un niño que vive en una familia muy controladora e invasiva, que critica y castiga todos sus intentos de autodefinición, puede ser protector y adaptativo comportarse de forma sumisa y desarrollar un estilo de apego aislado, ocultando su auténtico yo de manera autoprotectora, para evitar ser dañado.

El problema llega cuando el sujeto deja de ser consciente de la conexión de esos síntomas, de esas reacciones adaptativas, con el contexto inicial y de las funciones que cumplían y cumplen. Sacadas de contexto resultan incoherentes, extrañas y carentes de sentido. De manera que se podrían calificar de locas o patológicas.

Consecuentemente, la labor del psicoterapeuta será enfocar el tratamiento para facilitar:

a. Que sus clientes sean conscientes de los diversos significados de sus conductas, creencias y fantasías.

b. Que aprendan a reconocer y apreciar su función en un contexto y época determinada, así como su efecto en el presente, es decir, volver a encontrar la motivación arcaica y el hilo que une esa estrategia con el presente.

c. Normalizar sus comportamientos facilitando la comprensión de los contextos de los que derivan sus conductas, creencias o fantasías.

De esta manera, estará colaborando en qué diagnóstico y tratamiento, en interrelación, le proporcione al individuo una visión de sí mismo más normalizadora y humanizada.

El segundo principio filosófico, especialmente conectado con la diagnosis relacional es "Los seres humanos sufren perturbaciones relacionales, no "psicopatología".

En palabras de Erskine:

Una PI centrada en lo relacional enfatiza una perspectiva nopsicopatológica a la hora de entender la conducta de las personas. Los molestos síntomas fisiológicos y emocionales, los arraigados sistemas de creencias, las obsesiones y las conductas compulsivas, la agresión o el retraimiento social son todos ellos ejemplos de intentos creativos de satisfacer necesidades relacionales y de resolver interrupciones en el contacto interpersonal. (2016, p. xxxv)

Haciendo un paréntesis, una de las autoras comparte sus reflexiones a propósito de esta cita:

"Esta visión no patologizante resonaba con mi vivencia en el contacto directo con mis clientes y, a la vez, me confundía. Teniendo en cuenta que he desarrollado mi experiencia profesional en instituciones con un marco de referencia médico, en el que a los usuarios se les ve y se les define como enfermos, desde el supuesto conocimiento de los expertos profesionales. Y que, por otra parte, la mayoría de los clientes con los que he trabajado presentaban manifestaciones características 
de lo que clásicamente se denomina Trastorno Mental severo, tal y como recoge el Manual Diagnóstico y Estadístico de los Trastornos Mentales de la Asociación Americana de Psiquiatría (5 ed.; DSM-5):

Un Trastorno Mental es un síndrome caracterizado por una alteración clínicamente significativa, del estado cognitivo, la regulación emocional o el comportamiento del individuo, que refleja una disfunción de los procesos psicológicos, biológicos o del desarrollo, que subyacen en su función mental. Habitualmente los Trastornos mentales van asociados a un estrés significativo o a discapacidad, ya sea social, laboral o de otras actividades importantes. (p. 20)

Efectivamente, mis clientes podían ser diagnosticados como obsesivoscompulsivos, pasivo-agresivos, tener conductas manipuladoras, jugar a juegos psicológicos, podían estar fuera del contacto con la realidad...

Entonces me cuestionaba ¿cómo podía integrar mi marco de referencia médico con mi experiencia clínica de que, con frecuencia, mis prejuicios teóricos, el presuponer que sé lo que le pasa a mi cliente y el definirle anticipadamente con una categoría diagnóstica, más que ayudarme a conocerle y, sobre todo, más que ayudarle a hacerse consciente de lo que conoce de sí mismo, me inducía a error y me alejaba de una relación de pleno contacto con el yo auténtico de la persona que tenía enfrente?

Podía ser que la persona se adaptara y aceptara lo que yo dijera, pero que luego se escondiera (ocultara su yo real) y ya no hubiera una relación auténtica. $O$ que se rebelase y entrase en pelea, afectando igualmente a la alianza terapéutica.

Al mirar al otro como enfermo, como "patológico", perdía de vista la acomodación creativa, única de esa persona y sus intentos de manejarse en situaciones de negligencia, humillación y/o abuso, es decir, perdía la clave para comprender cómo el sujeto había llegado a esa situación, que condicionaba tanto su presente.

También perdía la oportunidad de establecer una relación interpersonal con un contacto auténtico. En el que apreciamos y valoramos la vulnerabilidad emocional del otro, sus necesidades relacionales y sus intentos desesperados de autorreparación, autorregulación o automejora, posibilitando la cura de heridas psicológicas arcaicas.

Me ayudó a encajar las piezas el enfocarme en explorar qué había más allá de la etiqueta diagnóstica, para poder mirar de frente al otro y verle como un ser humano valioso, único, vulnerable y con capacidad de desarrollar un sistema de afrontamiento creativo, que con frecuencia me maravillaba al descubrir cómo le ayudó a sobrevivir en un ambiente negligente o destructivo. Y me pregunto ¿cómo hubiera afrontado yo esa situación? Y ¿cómo estaría yo ahora si hubiera pasado por esa experiencia?

También fue clarificador la distinción entre los conceptos de Estilo-PatrónTrastorno. 


\section{Estilo - Patrón - Trastorno/Desorden}

"Un rasgo es una propiedad o peculiaridad distintiva", que permite identificar a algo o alguien (Pérez Porto y Merino, 2014). Las personas son seres complejos y, por tanto, tienen muchos rasgos comunes como especie, comunes a todos los seres humanos y es la combinación de estos rasgos unido a la intensidad de los mismos lo que les permite diferenciarse y marcar su identidad como individuo.

De manera que todos -por ser humanos- pueden ser perfeccionistas, obsesionarse, aislarse, mostrarse muy activos y expresivos emocionalmente... en alguna medida. Todas estas formas de sentirse y de mostrarse podrían representarse en un continuum (de menos a más) para ayudar a describir la intensidad con que un rasgo está presente en un sujeto en un momento dado o como característica general en su personalidad. Representado en un continuum de menor a mayor intensidad, que va desde la expresión leve, a otra moderada y a otra más severa, Erskine (2011, 2015/2016) describe tres categorías:

a) Estilo: cuando la persona manifiesta una serie de rasgos, que en mayor intensidad podrían describir una categoría diagnóstica, sólo cuando está bajo estrés o sienta amenazada su integridad física o psicológica por alguna razón (una pérdida, presión de las circunstancias vitales, exceso de intimidad, etc.). El resto del tiempo no resulta problemático para ella ni para otros. Por ejemplo, una persona con estilo borderline, como diría Erskine sería un “borderline en reserva” (Erskine, 2012), no todo el tiempo, pero sale de la reserva y lo actúa cuando aumenta la presión, el estrés... Se puede inferir el estilo de los clientes, cuando hablan de su sistema de afrontamiento en momentos de crisis, de sus sueños, de sus fantasías de futuro o cuando lo actúan sutilmente en transacciones transferenciales.

b) Patrón: cuando la persona repite una serie de rasgos de comportamiento y de relación de una manera predecible, no sólo en momentos de estrés, sino también en momentos o relaciones de mayor intimidad, como por ejemplo en familia. Puede que no lo actúe en terapia, o al menos inicialmente, hasta que la alianza y la percepción del terapeuta como alguien dependible esté más establecida. En todo caso, la familia y los más allegados pueden informar al respecto. Son precisamente los que le rodean, especialmente los más cercanos, los que suelen presentar las quejas, porque lo viven como algo incómodo, aunque frecuentemente el sujeto lo ve como algo normal. Así pues, representa un nivel más problemático de funcionamiento.

c) Trastorno / Desorden: cuando la persona actúa los rasgos en cualquier contexto, continuamente, no sólo bajo estrés o en intimidad e interfiere en casi todos los aspectos de su vida. Por eso, cuando el desorden es obvio, es fácil apreciarlo desde la primera o segunda sesión.

Son patrones de funcionamiento disfuncionales, que en algún contexto y momento tuvieron sentido y cumplieron alguna función protectora, pero que en la actualidad influyen e interfieren en las relaciones interpersonales que establece y 
en su proceso de desarrollo y autorrealización.

La evidencia de la gravedad del guion puede manifestarse a través del problema actual que presenta, a través de sus gestos físicos y en la reacción contratransferencial generada en el psicoterapeuta física y emocionalmente.

En este continuum, es el concepto de Trastorno / Desorden el que corresponde a las clasificaciones y descripciones clasificados y descritos en el CIE-10 y en el DSM-5.

\section{Fijaciones, Anhelos y Necesidades Arcaicas}

Quizá pueda traer algo de luz a este tema la reflexión sobre el proceso de formación de patrones relacionales fijados, en el contexto de la vivencia reiterada de fallos relacionales de figuras significativas.

Eric Berne (1973/1974)ya planteaba tres impulsos o apetitos humanos presentes en las personas a lo largo de toda su vida. Estos apetitos o impulsos los describe como la necesidad de estímulo o sensación, la necesidad de reconocimiento o relación y la necesidad de estructura o significado. La Psicoterapia Relacional Integrativa, recoge este planteamiento en su teoría de la motivación (O’Reilly-Knapp y Erskine, 2003), así como, en el principio filosófico "Todas las personas buscan relaciones y son interdependientes a lo largo de toda la vida” (Erskine, 2016). El contacto es el medio de satisfacer las necesidades relacionales. La relación, por consiguiente, se convierte en una fuente de motivación.

De la misma manera que nuestro cuerpo necesita nutrientes (proteínas, agua, vitaminas...) para que cada célula y cada órgano físico y psíquico funcione correctamente, también la relación (Erskine, 2011) puede aportar una serie de componentes o "nutrientes" indispensables para mantener la salud (seguridad, validación, aceptación, reciprocidad, autodefinición, experiencia de hacer impacto, iniciativa ajena, expresión de amor o gratitud). Estos nutrientes, en su conjunto, se denominan necesidades relacionales.

Cuando las relaciones interpersonales son de calidad, con un contacto interno y externo que permite satisfacer estas necesidades, el sujeto experimenta una vida satisfactoria, con sensación de plenitud de conexión interpersonal e integración personal.

Cuando las interrupciones del contacto impiden satisfacer algunas de estas necesidades, la calidad de la existencia se ve afectada. Si las interrupciones son frecuentes, el individuo pasa de vivir a sobrevivir y esto se manifiesta física, mental, emocional y conductualmente, en restricciones fisiológicas, desarrollo de creencias limitantes en relación a sí mismo, los otros, el mundo, patrones relacionales...

Tomando como referencia el ciclo gestáltico contacto-retirada (Perls, 1973/1976), se describe la dinámica de funcionamiento de las necesidades humanas, en este caso, de las necesidades relacionales:

1. Se inicia con la presencia de un estímulo que agita el organismo del individuo. 
2. Aparece la percepción de una necesidad, que pasa a primer plano.

3. Experimenta una incomodidad.

4. Busca la satisfacción manifestando ese malestar (llanto, solicitando presencia...).

5. Cuando hay un contacto externo que posibilita la satisfacción de esta necesidad, el sujeto integra la experiencia y esa necesidad pasa a $2^{\circ}$ plano, pasa al fondo y aparece otra necesidad, que se convierte en figura.

Cuando existe una interrupción del contacto, cuando ese contacto no se produce de acuerdo a las necesidades relacionales de la persona, entonces:

a) Las necesidades no son satisfechas.

b) Se mantienen en primer plano (en forma) y su organismo se mantiene alerta en la búsqueda de satisfacción, anhelando, deseando intensamente, una experiencia relacional reparadora que le permita integrarla y pasar a otra.

c) Como necesita dar sentido (estructura) a la experiencia del fallo relacional, para manejar la incomodidad generada por la insatisfacción de la necesidad, se produce una gestalt inacabada que presiona para completarse (Perls et al., 1994/2006), que podríamos entender como un cierre artificial o secundario. Entonces, elabora una serie de estrategias que le permiten cerrar artificialmente la gestalt -desarrollando, por ejemplo, patrones de relación, como puede ser un patrón de relación evitativo y se convence de que no necesita al otro; o se esconde y aísla... Este cierre artificial es la base de reacciones de supervivencia, conclusiones tempranas y decisiones de guion.

d) Pueden llegar a convertirse en patrones relacionales fijados, cuando se utilizan frecuentemente, que operan fuera de la consciencia del individuo, como patrones pre-reflexivos del self en relación: estilo de apego, autorregulación, necesidades relacionales, creencias de guion e introyección.

Los intensos afectos y necesidades pueden permanecer como memorias implícitas fijadas, hasta que las experiencias de cambio vital o una relación terapéutica eficaz faciliten la integración, tal y como describe Erskine refiriéndose al proceso terapéutico.

El objetivo se encuentra en la disolución de las defensas fijadas en la interrupción del contacto, que interfiere en la satisfacción de las necesidades presentes y en el pleno contacto con uno mismo y con los demás en la vida actual. Esto se logra, a menudo, trabajando dentro de la transferencia, para permitir que el conflicto intrapsíquico sea expresado dentro de la relación terapéutica y sea respondido con transacciones empáticas apropiadas. (Erskine, 2015/2016, p. 23) 


\section{Ajuste Creativo y Estrategias de Afrontamiento}

Si no hay patología ¿cómo entendemos las conductas y manifestaciones disfuncionales? La psicosis, la obsesión..., la enfermedad no existe, son conceptos abstractos, sin identidad propia. Lo que existen son las personas que manifiestan una serie de síntomas o reacciones defensivas, como parte de un sistema de afrontamiento, para gestionar las emociones y adaptarse a un entorno en el que no puede cubrir sus necesidades relacionales, ante los fracasos ocurridos en las relaciones de dependencia significativas a lo largo de la vida.

Reacciones que, con el paso del tiempo y el cambio de contexto relacional, se convierten en disfuncionales e incluso destructivas para sí mismo o para los que le rodean, al no actualizarse y ajustarse a las circunstancias presentes.

Algunos de estos intentos desesperados de autorregulación y ajuste creativo son: las interrupciones del contacto (como la negación, desapropiación, despersonalización, retroflexión, introyección, desapego emocional); las creencias de guion; los patrones de apego; y los guiones de vida.

\section{Diferencia entre un Diagnóstico Clásico y una Diagnosis Relacional}

A modo de resumen, en la tabla 1 se recogen algunas de las diferencias que se pueden observar entre el diagnóstico clásico y una diagnosis relacional.

Tabla 1. Diagnóstico Clásico versus Diagnosis Relacional

\begin{tabular}{|c|c|c|}
\hline & DIAGNÓSTICO CLÁSICO & DIAGNOSIS RELACIONAL \\
\hline Objeto & $\begin{array}{l}\text { La enfermedad } \\
\text { Clasificación de un síndrome } \\
\text { o cuadro psicológico }\end{array}$ & $\begin{array}{l}\text { La persona en su globalidad en } \\
\text { todas sus dimensiones }\end{array}$ \\
\hline ¿Qué destaca? & Lo patológico & $\begin{array}{l}\text { Los recursos creativos y } \\
\text { adaptativos del sujeto en } \\
\text { contextos problemáticos }\end{array}$ \\
\hline \multirow[t]{2}{*}{$\begin{array}{l}\text { ¿A quién } \\
\text { empodera? }\end{array}$} & $\begin{array}{l}\text { Al terapeuta } \\
\text { Experto en la solución }\end{array}$ & $\begin{array}{l}\text { Al cliente } \\
\text { (Sintonía rítmica, un paso por } \\
\text { detrás del cliente) }\end{array}$ \\
\hline & Establecido unilateralmente & Co-creado \\
\hline Enfoque & $\begin{array}{l}\text { En las manifestaciones o } \\
\text { síntomas presentes }\end{array}$ & Mirada evolutiva, procesual \\
\hline \multirow[t]{2}{*}{ Metodología } & Interpretación & Indagación \\
\hline & $\begin{array}{l}\text { Clasifica clichés en } \\
\text { categorías generales }\end{array}$ & $\begin{array}{l}\text { Cuenta una historia descriptiva y } \\
\text { personalizada }\end{array}$ \\
\hline $\begin{array}{l}\text { Tipo de } \\
\text { Procedimiento } \\
\text { y Tratamiento }\end{array}$ & $\begin{array}{l}\text { Ofrece procedimientos } \\
\text { standares para cada } \\
\text { diagnóstico } \\
\text { Soluciones y recetas } \\
\text { externas }\end{array}$ & $\begin{array}{l}\text { Proceso no standard. Terapia } \\
\text { relacional: dos personas uniendo } \\
\text { sus personalidades para co-crear } \\
\text { un proceso de sanación interna, } \\
\text { desde una relación de pleno } \\
\text { contacto }\end{array}$ \\
\hline
\end{tabular}




\section{Diagnóstico Relacional Integrativo en Acción}

El marco teórico de la Psicoterapia Relacional Integrativa sirve de guía, a modo de mapa conceptual, desde donde moverse a la hora de hacer psicoterapia.

El diagnóstico, desde este modelo, se podría definir como Diagnosis Relacional y se caracteriza, por ser un proceso de evaluación de la personalidad y del sistema de afrontamiento y adaptación de una persona, para identificar pautas de funcionamiento (afectivo, cognitivo, fisiológico y conductual) y patrones relacionales inconscientes, a veces disfuncionales, fijados en etapas evolutivas en que tuvieron lugar rupturas de contacto con personas significativas, que subyacen al motivo de consulta que la persona trae consigo con la finalidad de alcanzar un objetivo.

Es un proceso de evaluación dinámico, que se desarrolla a lo largo de todo el proceso terapéutico, a modo de diferentes aproximaciones cada vez más precisas, en la medida en que se va recopilando información y confirmando o descartando hipótesis en el marco de la relación co-creada entre psicoterapeuta y cliente.

Por lo tanto, de la misma manera que se diferencia entre un contrato inicial y contratos de proceso, también se puede distinguir entre un diagnóstico inicial, que es con frecuencia a lo que se refieren en la literatura cuando hablan de hacer un diagnóstico, y diagnóstico de proceso.

Se trata de un proceso de recogida de información en la relación, a través de la indagación sintónica en la que el terapeuta va a seguir al cliente, va ir por detrás de la persona, respetando su ritmo, para que vaya descubriendo y dándose cuenta por sí misma, de la información que va emergiendo a través de las indagaciones del terapeuta. En algún momento tal vez se le ofrezca un resumen recopilatorio, una devolución de la información, que ha ido surgiendo, para facilitarle una visión de conjunto o para subrayar lo que han ido descubriendo en diferentes sesiones y que eso le sirva para seguir avanzando. Entonces, de alguna manera, se combinan contrato y diagnóstico de proceso, con tratamiento.

Tomando como referencia los diferentes esquemas elaborados por representantes de la Psicoterapia Relacional Integrativa, para explicar las teorías de la personalidad y los métodos desde esta corriente, y el continuum entre Estilo - Patrón - Trastorno /Desorden (que aporta la diferencia entre normalidad y trastorno, fundamentada en nociones cuantitativas más que cualitativas) se irán recopilando los datos para realizar el psicodiagnóstico del cliente (figura 1).

Así pues, cuando alguien viene a consulta, el terapeuta empieza a establecer una relación en la que se compromete con el bienestar del cliente, escuchando lo que éste desea y necesita del terapeuta y de la relación. Y comienza la evaluación (desde las primeras impresiones, incluso desde la primera toma de contacto antes de venir), con la intención de entender al cliente e identificar los "asuntos clave" (Clarkson, 1992, citada en Mazzetti, 2012) que necesita y/o desea trabajar, para finalmente valorar si puede ofrecerle ayuda y en qué modo.

La relación co-creada va a ser la base desde la que hará la diagnosis, con la información que progresivamente vaya facilitando y facilitándose, tomando 
Figura 1. Desarrollo de la Diagnosis Relacional

ESQUEMA DEL DESARROLLO DE LA DIAGNOSIS RELACIONAL ¿Cómo?

EL SEUF EN RELACIÓN

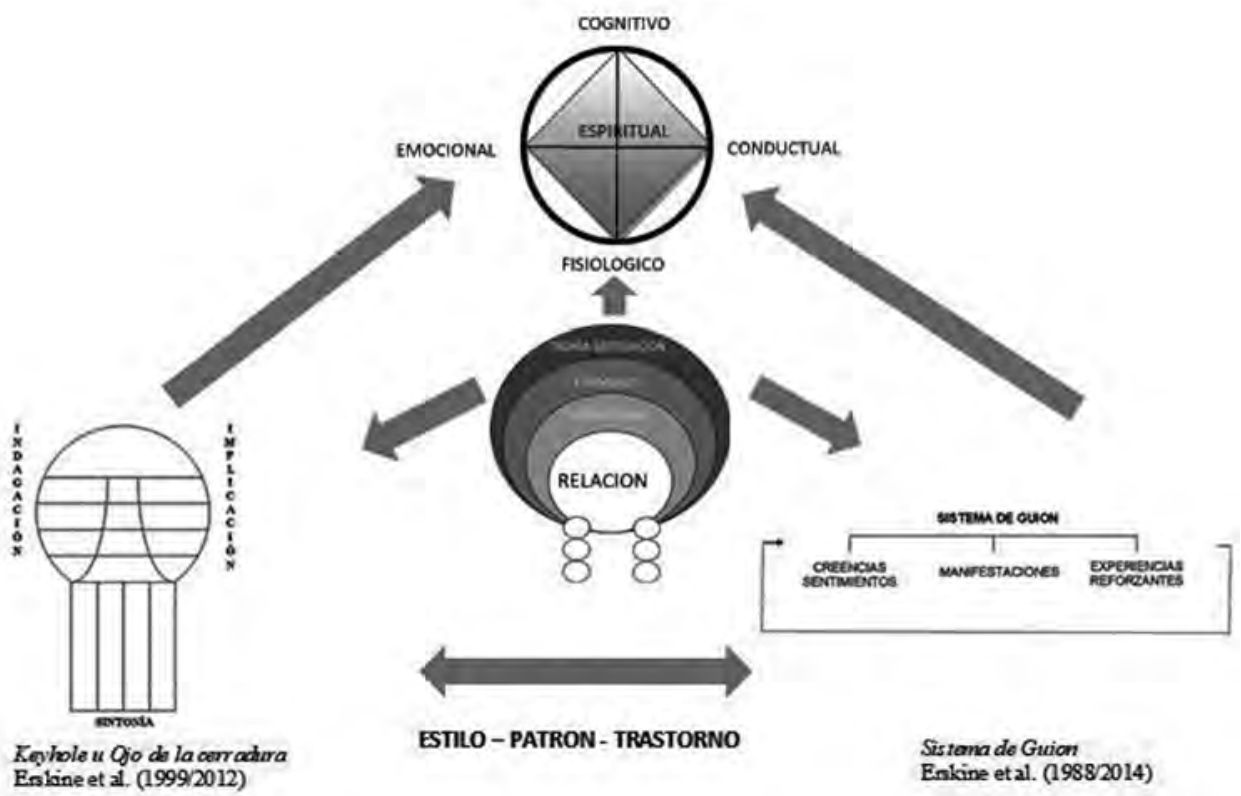

conciencia el cliente, a medida que se va estableciendo la alianza terapéutica, la seguridad y confianza necesaria para que la persona se atreva a enfrentarse a sus miedos, dificultades y explorar aspectos de sí que, por alguna razón, mantiene fuera de la consciencia. Es decir, crear una relación terapéutica lo suficientemente confiable y segura para poder acompañar a la persona cogida de la mano, a esos lugares oscuros que forman parte de su historia.

Gracias a esta dinámica de interacción intersubjetiva, co-creada y única con cada cliente, éste va a ir ampliando su autoconocimiento en todas sus dimensiones (esquema del self en relación, figura 1) fisiológica, afectiva, cognitiva, conductual y espiritual y, el terapeuta tomará nota de la calidad del contacto y el tipo de apego que presenta. Lo cual le va a permitir ir haciendo hipótesis que, en su momento, irá corroborando o descartando.

Describirá el efecto en el presente, a nivel interno y externo, de las experiencias traumáticas (tanto agudas como acumulativas), que el cliente ha ido atravesando a lo largo de su vida como consecuencia de fallos relacionales con figuras significativas.

Las autoras prefieren utilizar el término de "impresión diagnóstica", porque se ofrece al cliente la oportunidad de aceptarlo o no y, sobre todo, entrar en un diálogo con sus matices, contrastes...

El terapeuta, sirviéndose del diagrama del Keyhole (figura 1), utilizará los 
diversos tipos de indagación para facilitar al cliente su autoconocimiento y la integración de los aspectos escindidos de sí, a la vez que va conociéndole como objetivo secundario.

En este punto describirá:

a) su experiencia fenomenológica (emociones, sensaciones...)

b) cómo elabora el sentido de su experiencia vital, que le orientará sobre lo que necesita para construir una narrativa histórica integrada. Así como, el o los contextos iniciales que dieron lugar a la elaboración del guion de vida (origen histórico)

c) sus estrategias de afrontamiento (apreciando los recursos disponibles)

d) su experiencia de la relación transferencial y la contratransferencia elicitada

Para guiarse en la indagación, buscará mantenerse en sintonía y prestar atención:

a) a las necesidades relacionales que presenta en cada momento;

b) a su ritmo de elaboración y procesamiento de las experiencias;

c) a sus afectos;

d) a su forma de dar sentido y crear significados desde su marco de referencia cognitivo;

e) a la etapa del desarrollo evolutivo en la que se manifiesta en cada momento.

El Esquema del Sistema del Guion (figura 1) es otro poderoso elemento de autoconocimiento, al representar la organización inconsciente de la experiencia del sujeto, además de ser una herramienta útil de diagnóstico y tratamiento en el trabajo psicoterapéutico profundo. Provee al terapeuta de comprensión, impresión diagnóstica y le aporta una dirección de tratamiento.

Enfocándose en este esquema, desde las manifestaciones del guion, teniendo en cuenta que en los síntomas está encriptada una historia que tiene un significado y un sentido (tanto en el presente, como en el pasado), intentará llegar a las creencias de guion (sobre sí mismo, sobre los otros y sobre la cualidad de la vida o el mundo) que subyacen a esas manifestaciones y que influyen selectivamente en la forma de percibir, en la manera de dar significado a la experiencia y en los sentimientos generados.

A través del trabajo con las manifestaciones y las creencias de guion puede acceder también a los sentimientos reprimidos y las necesidades no cubiertas, en el tiempo de la formación del guion de vida.

Pero la impresión diagnóstica no será completa hasta no identificar cuáles son las experiencias reforzantes que mantienen activo el guion y resistente al cambio de las creencias, las manifestaciones y los afectos. Especialmente, identificar las funciones psicológicas específicas para este individuo. Finalmente, será relevante considerar el grado de afectación, el nivel más o menos significativo de malestar, interferencia y disfuncionalidad, así como la cantidad de áreas de su vida que se encuentran afectadas.

$\mathrm{Y}$ es en esta manera interactiva en que contrato, diagnosis relacional y tratamiento psicoterapéutico, se van entrelazando de forma dinámica, tejiendo un tapiz: el 
diagnóstico orienta el plan de tratamiento y, a su vez, cada intervención terapéutica aporta relevante información para refinar el diagnóstico y ajustar el tratamiento, todo ello enmarcado en los límites del contrato terapéutico, que otorga protección y seguridad a ambas partes implicadas.

El objetivo final sería elaborar de forma conjunta con el protagonista, una imagen diagnóstica descriptiva (global) del cliente y su contexto presente y pasado. Este proceso queda representado en la figura 2.

Figura 2. Diagnosis Relacional

1. Asuntos Clave: ¿Que viene buscando?

2. Descripción del sujeto desde todas sus

Calidad del contacto (puerta abierta al contacto) dimensiones. ¿A quién tenemos delante?

- Tipo de apego . Necesidades Relacionales

- Imagen evolutiva . Experiencia fenomenológica

3. Efecto actual, a nivel interno y externo, de experiencias vitales traumäticas

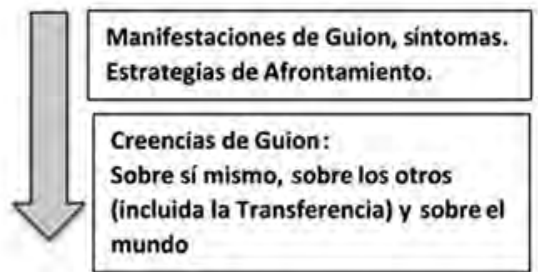

4. Grado de Afectación

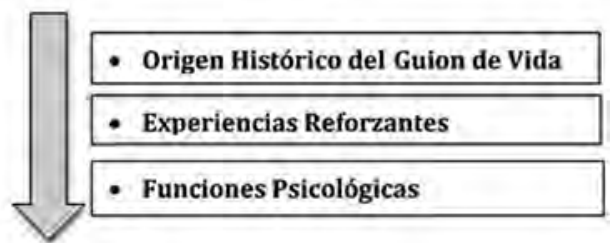

Nivel más o menos significativo de malestar, interferencia, disfuncionalidad y cantidad de áreas afectadas.

\section{Caso Jota}

Presentamos a continuación el caso de Jota como ilustración de la diagnosis relacional. Aunque por motivos de espacionolo haremos de una manera exhaustiva.

Jota es un varón de 47 años. Soltero. Vive solo en la ciudad. Trabaja como funcionario. Es el $3^{\circ}$ de 4 hermanos.

Acude a consulta por sus dificultades de relación, especialmente con las mujeres. Presenta diversas quejas y manifestaciones somáticas (insomnio, cistitis recurrentes...). Lleva 10 años en tratamiento psicofarmacológico (ansiolítico y antidepresivo) y terapéutico con un médico-psiquiatra. Relata que no ha conseguido los cambios que él esperaba. "El psiquiatra me dice lo que tengo que hacer, pero no me funciona". No sabe identificar lo que espera del tratamiento, pero sí manifiesta su anhelo de tener su propia familia. Acordamos, como contrato psicoterapéutico, trabajar juntos enfocados en el objetivo de conocerse y entenderse mejor, comprender el posible significado de sus manifestaciones somáticas, asi como sus dificultades de relación para, de ese modo, ser más consciente de sí mismo y de sus relaciones y poder elegir el manejo de las diferentes situaciones. 


\section{Información Recogida}

En el desarrollo de las sesiones, se observa, la dificultad de Jota en el contacto visual sostenido, y su tensión corporal. Habla de sí mismo en segunda y tercera persona, muestra dificultad para identificar su proceso interno, especialmente, su mundo emocional. Parece abierto al contacto en la dimensión cognitiva. Percibe al terapeuta con ambivalencia, por una parte, espera que se le diga qué tiene que hacer, y por otra, con desconfianza, “yo no creo en estas cosas” refiriéndose a la psicoterapia, con un tono arrogante y desde una postura de superioridad.

Relata una vida solitaria, centrada en el estudio y en su familia de origen. Tiene muy pocas relaciones, que describe con poco contacto interpersonal "yo mantengo las distancias”. Pasa la mayor parte del tiempo en que no está trabajando, centrado en el estudio, y visitando a su familia de origen que vive en otra localidad.

Mientras vamos tejiendo la relación terapéutica, apreciamos que en el relato de su historia falta el recuerdo de interacciones de dependencia con sus cuidadores. en su infancia, niñez y adolescencia. Recuerda un ambiente familiar de mucha tensión y muchos reproches "callados” entre los padres. Tanto el padre como la madre no estaban disponibles emocionalmente, incómodos al contacto físico. Jota no encontraba una respuesta emocional, ni el contacto que necesitaba en ellos. Sólo conseguía sentirse visto cuando presentaba, en casa, el resultado de sus estudios. Sin embargo, justifica y protege a sus progenitores.

En la actualidad, aunque expresa su deseo de relación, devalúa la importancia de las relaciones, mostrándose frío, anticipando el rechazo por parte de los demás, suele enfadarse e indignarse con las personas con las que se relaciona evitando la intimidad. Llena el vacío de soledad con preocupación y fantasías repetitivas. Para calmar el dolor, puede oscilar entre el autoengrandecimiento y la autodevaluación. Teme mostrarse necesitado y vulnerable.

\section{Impresión Diagnóstica}

Podemos identificar en Jota las manifestaciones del trauma acumulativo, que tiene su origen en esa falta de unos progenitores disponibles, demasiado inmersos en su propio conflicto e incómodos con el contacto físico, para acompañarlo, calmarlo, ayudarle a identificar su mundo interno de sensaciones y emociones, y poder articularlo en pensamientos, palabras, en acciones, todo ello dentro de una relación sólida y confiable.

Esta falta de contacto no permitió que Jota pudiera cubrir sus necesidades relacionales de seguridad, ser validado, autodefinirse, hacer un impacto en los otros. Y también, llevó a mantener fuera de su conciencia, el enfado, la tristeza, el dolor y la vitalidad, lo que puede conducir a la hipótesis de una posible depresión subyacente.

Como resultado, presenta un patrón de apego inseguro, de tipo evitativo. Un desapego emocional, que cognitivamente justifica con las creencias "estar cerca es peligroso", "los otros me rechazarán”, "la vida es peligrosa”. Y una 
percepción de sí mismo de que "no importo”, “algo está mal en mí”. Creencias que se ven reforzadas por sus fantasías y que condujeron a la decisión temprana de "lo haré solo".

Asimismo, Jota muestra un patrón obsesivo, con el que llena el vacío y la soledad con preocupación y fantasías repetitivas. Unido a un patrón narcisista, para calmar su dolor emocional, oscilando entre el autoengrandecimiento y la autodevaluación.

Durante el proceso terapéutico, en ocasiones se muestra como un niño de 8 años, con un tipo de pensamiento concreto, que busca ser visto y valorado a través del estudio, y en otras ocasiones, la imagen evolutiva que muestra es de un niño de 2 años aislado y solo, anhelando contacto y calidez.

Jota ha ido desarrollando, en soledad, estrategias de afrontamiento, para manejar todo aquello que le estaba ocurriendo; aprendió a tensar sus músculos para no sentir (retroflexión); a permanecer en aislamiento social evitando la intimidad y las relaciones en general, por temor al rechazo anticipado y a la vulnerabilidad; a desapegarse emocionalmente para no sentir el dolor de no sentirse visto, ni valorado, y manejar también su soledad; y a repetir internamente, esas creencias en relación a sí mismo, los otros y el mundo. Refugiándose en fantasías de ser envidiable, así como fantasías de venganza y castigo a otros.

Como experiencias reforzantes encontramos: falta de relaciones de intimidad. En el trabajo, espera un ascenso y se lo dan a otro compañero. Viejas memorias emocionales: el recuerdo del sentimiento de soledad en brazos de su madre. En la adolescencia, el mensaje de la madre: "cualquier palabra o acción tuya se puede convertir en un daño para ti"; y el mensaje del padre: "calla y piensa sobre lo que pasa”.

Entre las funciones psicológicas de su conducta y sus manifestaciones de guion, observamos el uso del estudio y el aislamiento como una manera de autoestabilización, regulación y reparación.

Apreciamos una afectación significativa en el área personal, en la relación consigo mismo y con los demás, que condiciona su día a día, resultando el área profesional la más conservada.

Una vez presentado el caso desde la diagnosis relacional, pasamos a presentar el diagnóstico de Jota desde el diagnóstico clásico, Manual diagnóstico y estadístico de los trastornos mentales. (DSM-5)

Eje I $\quad$ F41.1 Trastorno de ansiedad generalizada [300.02]

Eje II F60.5 Trastorno obsesivo-compulsivo de la personalidad [301.4]

F60.81 Trastorno narcisista de la personalidad [301.81]

Frecuente uso de la negación y la idealización

Eje III Ninguno

Eje IV Ninguno

Eje V EEAG=55

Después de la exposición de estos dos procedimientos diagnósticos, podemos 
apreciar la diferencia entre el grado de conocimiento de Jota, tanto para sí mismo como para el terapeuta.

Comparativamente, el diagnóstico clásico desde DSM-5 y CIE-10 es más sintético y rápido, mientras que desde la Psicoterapia Relacional Integrativa es más extenso, pero a la vez más descriptivo, personalizado y concreto. Desde el diagnóstico clásico, tenemos una visión muy general de una tipología de personas, con unas características comunes. En la diagnosis relacional, podemos ver a la persona, tenemos esa imagen tridimensional y holográfica del cliente, un cuadro impresionista que nos permite ver a través de las pinceladas, la persona en su conjunto, en acción dinámica y evolutiva, en el contexto en que se desenvuelve.

\section{Conclusión}

Como conclusión de este trabajo se define la evaluación clínica como un proceso dinámico y continuo, dado que la persona y sus circunstancias son cambiantes, van evolucionando. El terapeuta se encontrará “evaluando” (recogiendo información, haciendo y contrastando hipótesis...) a lo largo de todo el proceso de tratamiento y de la relación terapéutica.

Contrato, diagnosis relacional y tratamiento psicoterapéutico, se van entrelazando de forma dinámica e interactiva: el diagnóstico orienta el plan de tratamiento y, a su vez, cada intervención terapéutica aporta relevante información para refinar el diagnóstico y ajustar el tratamiento, todo ello enmarcado en los límites del contrato terapéutico, que otorga protección y seguridad a ambas partes implicadas.

El objetivo final sería elaborar de forma conjunta con el protagonista, una imagen diagnóstica descriptiva, global, del cliente y su contexto presente y pasado.

El diagnóstico es inevitable. El interrogante es cómo realizarlo. Y siempre produce un efecto, en la persona que lo recibe, dependiendo del significado que le dé.

En este artículo se apuesta por la coherencia entre transdiagnóstico, transteoría y transmetodología de la Psicoterapia Relacional Integrativa.

Teniendo en cuenta los principios filosóficos, el psicodiagnóstico en Psicoterapia Relacional Integrativa debiera ser un proceso igualitario, no un acto de poder, enfocado en la globalidad de la persona, con una perspectiva evolutiva, prestando atención a las rupturas del contacto interno y externo y a la cantidad y la cualidad de las relaciones interpersonales que mantiene, de forma orientativa, evitando confundir a la persona con la etiqueta diagnóstica.

Es desde esta perspectiva que podemos ir comprendiendo la personalidad, el sistema de afrontamiento, los patrones relacionales inconscientes y las etapas evolutivas en que se desarrollaron. 


\section{Referencias}

American Psychiatric Association (2014). Manual diagnóstico y estadístico de los trastornos mentales (5 ed.). Editorial Médica Panamericana.

Berne, E. (1974). ¿Qué dice Usted después de decir Hola? (N. Daurella, Trad.). Grijalbo. (Trabajo original publicado en 1973)

Diagnóstico (n.d.). En Significados.com. Recuperado el 14 de agosto de 2020 https://www.significados.com/ diagnostico/

Erskine, R. G. (1997). Theories and Methods of an Integrative Transactional Analysis: A Volume of Selected Articles [Teorías y métodos del análisis transactional integrativo: un volumen de artículos seleccionados]. TA.

Erskine, R. G. (2010). Life Scripts: A Transactional Analysis of Unconscious Relational Patterns [Guiones de vida: un análisis transactional de patrones relacionales inconscientes]. Karnac Books.

Erskine, R. G. (febrero 2011). Trastornos del apego, Patrones Relacionales e Implicación Terapéutica. [Seminario presencial] Instituto Bios de Psicoterapia Integrativa, Murguía. Álava, España.

Erskine, R. G. (2015). Transactional Analysis in Contemporary Psychotherapy. Karnac Books.

Erskine, R. G. (2016). Presencia terapéutica, patrones relacionales: conceptos y práctica de la Psicoterapia Integrativa (A. Pérez Burgos, Trad.). Karnac Books. (Trabajo original publicado en 2015)

Erskine, R. G. y Moursund, J. P. (2014). La Psicoterapia Integrativa en Acción (I. Arregui, Trad.). Desclée de Brouwer. (Trabajo original publicado en 1988)

Erskine, R. G., Moursund, J. P. y Trautmann, R. L. (2012). Más allá de la empatía: una Terapia de Contacto-enla-Relación (N. Mestre, Trad.). Desclée de Brouwer. (Trabajo original publicado en 1999)

Erskine, R. G. (septiembre, 2012). El tratamiento de las personalidades borderlines. El paciente borderline, la confusión temprana del afecto [Seminario]. Vilanova Barcelona, España.

Fagan, J. (1973). Las tareas del terapeuta. En J. Fagan y I. Sheperd (Eds.), Teoría y técnica de la psicoterapia guestáltica (pp. 93-111). Amorrortu.

Gimeno-Bayón, A. y Rosal, R. (2001). Psicoterapia Integradora Humanista. Manual para el tratamiento de 33 problemas psicosensoriales, cognitivos y emocionales. Desclée de Brouwer.

Gimeno-Bayón, A. (2012). Comprendiendo la Psicoterapia de la Gestalt. Milenio.

Linares, J. L., Castelló, N. y Colilles, M. (2001). La terapia familiar de las psicosis como un proceso de reconfirmación. Revista Redes, 8, 9-29. http://redesdigital.com.mx/index.php/redes/article/view/121/60

Livesley, W. J. (2018). Terapia transdiagnóstica y transteórica para el trastorno de personalidad. Revista de Psicoterapia, 29(110), 89-110. https://doi.org/10.33898/rdp.v29i110.249

Mazzetti, M. (2012). Supervisione in Analisi Transazionale. Rivista Italiana di Analisi Transazionale, 25(62), 1-24. http://rivista.simpat.org/dett_articolo.php?id_articolo=738

Mirapeix, C. (2018). Un modelo transdiagnóstico guiado por el Self: Una visión ampliada de la psicoterapia cognitivo analítica. Revista de Psicoterapia, 29(100), 111-133. https://oi.org/10.33898/rdp.v29i110.250

Moursund, J. P. y Erskine, R. G. (2004). Integrative Psychotherapy: The Art and Science of Relationship. Thomson: Brooks/Cole.

O’Reilly-Knapp M. y Erskine R. G. (2003). Core concepts of an Integrative Transactional Analysis. Transactional Analysis Journal, 33(2), 168-177. https://doi.org/10.1177/036215370303300208

Organización Mundial de la Salud (2010). Clasificación Estadística Internacional de Enfermedades y Problemas Relacionados con la Salud (10 ed.). Ministerio de Sanidad de España.

Pérez Porto, J. y Merino, M. (2014). Rasgo. En Definición.de. Recuperado (FECHA) https://definicion.de/rasgo/

Perls, F. (1976). El enfoque gestáltico. Testimonios de terapia (F. Huneeus, Trad.). Cuatro Vientos. (Trabajo original publicado en 1973)

Perls, F., Hefferline, R. F. y Goodman P. (2006). Terapia Gestalt: Excitación y crecimiento de la personalidad humana (C. Vázquez Bandin, M. C. García de Enterria, Trad.). Sociedad de cultura Valle-Inclán. (Trabajo original publicado en 1994)

Psicodiagnóstico (15 agosto 2020). En Wikipedia. https://es.wikipedia.org/wiki/Psicodiagn\%C3\%B3stico 